\title{
Dispersed phase mixing effects on second moments in dominantly first-order, backmix reactors
}

\author{
R. L. CURL \\ University of Michigan, Ann Arbor, Michigan \\ (Received 27 June 1966; accepted 29 July 1966)
}

\begin{abstract}
An idealized model for dispersed phase mixing is used to find the relative rates of $A+A$, $B+B$ and $A+B$ reactions when the reaction $A \rightleftharpoons B$ is dominant, first order and reversible. A large possible effect of mixing on the $A+A$ reaction is demonstrated, while the effect on $B+B$ on $A+B$ is never greater than a factor of two.

The results are applicable to estimating the effect of mixing on second order by-product reactions which are important when present to a small extent. In addition, this represents the first analytical solution to the mixing-reaction equation.
\end{abstract}

THE DIRECTION and magnitude of the effect of dispersed phase mixing on the conversion in systems with reaction within the dispersed phase has now been calculated for zero and second order reactions (CURL [1]; SPIELMAN and LEVENSPIEL [2]), using an idealized mixing model. These and related works on segregation in chemical reactors are reviewed by RIETEMA [3].

The idealized mixing model assumes that all drops are the same size, that coalescence occurs at random, and that after coalescence the newly formed drop is completely mixed and breaks immediately into two drops of the same size. While admittedly unrealistic, this simple model is all we have until something is learned of the details of the coalescence and breaking processes in reactors. In the meantime the idealized model has probably provided semiquantitative answers to several questions about the performance of dispersed phase reactors.

The Monte Carlo calculation procedure of Spielman and Levenspiel is admirably suited for computationally modelling more complex systems. Therefore there exists a relatively simple calculation procedure for obtaining numerical answers to as many more mixing-reaction systems as we can imagine.

Nevertheless there still remain reasons for being interested in possible analytical solutions of the equations describing the system. These may not only clarify the interaction of the parameters in the mixing-reaction models but also provide a more direct or simpler calculation of numerical results.
The general mixing-reaction equation (CURL [1]) is:

$$
\begin{aligned}
\frac{\partial p}{\omega_{r} \partial t} & =p_{0}-p+I\left[2 \int_{0}^{\infty} p(2 c-\alpha) p(\alpha) d \alpha-p\right] \\
& +K \frac{\partial c^{n} p}{\partial c}
\end{aligned}
$$

for the probability density distribution, $p(c, t)$ of drop concentration. This has no known analytical solution. The difficulty associated with solving it is illustrated by converting it to the $r$ th (integer) moments $\left(m_{r}\right)$ of $p$, giving

$$
\begin{aligned}
\frac{\partial m_{r}}{\omega_{r} \partial t} & =m_{r(0)}-m_{r} \\
& +I\left[\frac{1}{2^{r}} \sum_{k=0}^{r}\left(\begin{array}{l}
r \\
k
\end{array}\right) m_{r-k} m_{k}-m_{r}\right]-K r m_{r+n-1}
\end{aligned}
$$

(If $r+n-1=0, m_{\mathrm{o}}$ must exclude the area under any singularity at the origin and need not equal 1.0.) For $n \neq 1$ there is no finite (closed) set of simultaneous equations allowing the calculation of the individual $m_{r}$. Either equations for always higher moments, or endless fractional moments, are required. HulberT and KATZ [4] point out the possibility of closing or truncating the infiite set of equations by approximating the distribution $p$ with a series of special functions. This has not yet been attempted for this mixing-reaction model. Here the interest is in eludicating an analytical solution to Eq. (2). In particular, we see that we may obtain all the moments if $n=1$, although this is the one case where dispersed phase mixing does not affect the 
reactant conversion (RIETEMA [3]). However, there is a practical situation in which we are interested in the higher moments of the distribution of concentration in the mixing-first order reaction situation. That this also yields analytical results is a benefit in further contemplation of related problems.

Consider a reversible first order chemical reaction $\mathbf{A} \rightleftharpoons \mathbf{B}$ taking place in the drops in a dispersed phase, backmix reactor. The mean concentration of $A$, $\overline{C_{A}}$, will depend only upon the dispersed phase residence time and the kinetic rate constants. However, let us worry about the possibility of the second order reactions $\mathbf{A}+\mathbf{A}, \mathbf{B}+\mathbf{B}$ or $\mathbf{A}+\mathbf{B}$ going to some product which is undesirable in even trace quantities. The average rates of these reactions depend on the quantities $\overline{C_{A}^{2}}, \overline{C_{B}^{2}}$ and $\overline{C_{A} C_{B}}$ in the reactor, but these in turn depend on the rate of dispersed phase mixing. Hence, if we neglect the contribution of these reactions to the mass-balance in the reactor, we have a readily solvable problem, and can obtain analytical expressions for the extent to which dispersed phase mixing affects the relative rate of these possible by-product reactions.

For simplicity consider that only $\mathbf{A}$ is fed in the dispersed phase, at concentration $C_{A O}$. Stoichiometry requires that, due to reaction alone, the concentrations of $A$ and $B$ are related by

$$
C_{B}=C_{A O}-C_{A}
$$

Now, dispersed phase mixing, with the simple model of CURL [1], produces drops having the average concentrations of the species in the mixing drops. Hence the drops resulting from mixing must also have $C_{A}$ and $C_{B}$ as given by Eq. (3). This is shown in Fig. 1, where it is apparent that mixing of two

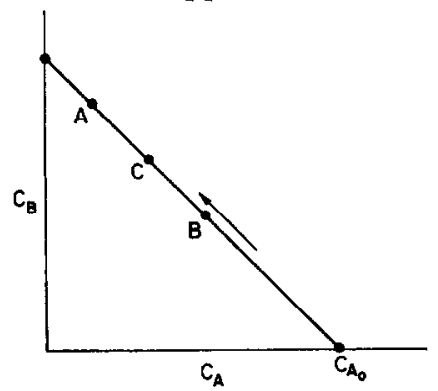

Fig. 1. The locus of all possible drop concentrations in the present system. The coalescence of a drop from $P t A$ with one from $P t B$ simply produces two at $P t C$. The arrow indicates the direction of drop "motion" due to reaction. drops from different locations on the line produces a concentration pair lying on the line. Therefore, even though we might have thought that the problem would have to be developed in terms of the joint probability density distribution $p\left(C_{A}, C_{B}\right)$, the dependence between $C_{A}$ and $C_{B}$ reduces the problem to one dimension. It is convenient to non-dimensionalize concentration by setting $y=C_{A} / C_{A O}$. In the one dimensional reaction (or "phase") space of $y$, the reaction velocity is, using first order kinetics and Eq. (3),

$$
\frac{d y}{d t}=k_{2}-\left(k_{1}+k_{2}\right) y
$$

The conservation equation, analogous to Eq. (1) is, in the steady state:

$$
\begin{aligned}
p_{\mathrm{o}}-p+I\left[2 \int_{0}^{\infty} p(2 y-\alpha) p(\alpha) d \alpha-p\right] \\
-\frac{\partial}{\partial y}\left(k_{2}-\left(k_{1}+k_{2}\right) y\right) p=0
\end{aligned}
$$

(The reaction term is conveniently obtained from the conservation, or "Liouville" equation, such as shown by HulbuRT and KATZ [4]). The reaction moduli $K_{1}$ and $K_{2}$, equal to $k_{1} / \omega_{r}$ and $k_{2} / \omega_{r}$, are for the forward and reverse reaction respectively.

The associated moment equation is:

$$
\begin{aligned}
m_{r_{\mathrm{o}}}-m_{r}-I & {\left[\frac{1}{2^{r}} \sum_{k=0}^{r}\left(\begin{array}{l}
r \\
k
\end{array}\right) m_{r-k} m_{k}-m_{r}\right] } \\
& -r\left[\left(K_{1}+K_{2}\right) m_{r}-K_{2} m_{r-1}\right]=0
\end{aligned}
$$

We immediately obtain (since $m_{o}=1$ and $m_{r_{\mathrm{o}}}$ has been assumed to equal $1 \cdot 0)$ :

$$
\begin{aligned}
& m_{1}=\frac{1+K_{2}}{1+K_{1}+K_{2}} \\
& m_{2}=\frac{1+2 K_{2}+m_{1}{ }^{2}(I / 2)}{1+2\left(K_{1}+K_{2}\right)+(I / 2)}
\end{aligned}
$$

We seek the means $\overline{C_{A}{ }^{2}}, \overline{C_{B}{ }^{2}}$ and $\overline{C_{A} C_{B}}$. In dimensionless form, using a joint moment notation, these are, respectively:

$$
m_{20}=\overline{y^{2}}=m_{2}
$$


Dispersed phase mixing effects on second moments in dominantly first-order, backmix reactors

$$
\begin{aligned}
& m_{02}=\overline{(1-y)^{2}}=1-2 m_{1}+m_{2} \\
& m_{11}=\overline{y(1-y)}=m_{1}-m_{2}
\end{aligned}
$$

while

$$
\begin{aligned}
& m_{10}=\bar{y}=m_{1} \\
& m_{01}=1-\bar{y}=1-m_{1}
\end{aligned}
$$

With $I \rightarrow \infty$, the well mixed dispersed phase situation, the concentrations are uniform throughout and we would have

$$
\begin{aligned}
& m_{20}=m_{10}{ }^{2} \\
& m_{02}=m_{01}{ }^{2} \\
& m_{11}=m_{10} m_{01}
\end{aligned}
$$

The relative rates of the trace second order reactions, with respect to the well mixed case, are then

$$
\begin{aligned}
& \gamma_{20}=\frac{\overline{C_{A}^{2}}}{\overline{C_{A}}}=\frac{m_{20}}{m_{10}{ }^{2}} \\
& \gamma_{02}=\frac{\overline{C_{B}^{2}}}{\overline{C_{B}^{2}}}=\frac{m_{02}}{m_{01}{ }^{2}} \\
& \gamma_{11}=\frac{\overline{C_{A} C_{B}}}{{\overline{C_{A}}}_{A} \bar{C}_{B}}=\frac{m_{11}}{m_{10} m_{01}}
\end{aligned}
$$

In place of the reaction moduli, the following variables are chosen to represent the reaction conditions:

1. Possible equilibrium conversion (from 7 and $\left.13, K_{1,2} \rightarrow \infty\right)$

$$
E=m_{01 \mathrm{e}}=\frac{K_{1}}{K_{1}+K_{2}}
$$

2. Fractional approach to equilibrium (from 7, 13 and 20)

$$
A=\frac{m_{01}}{m_{01 \mathrm{e}}}=\frac{K_{1}+K_{2}}{1+K_{1}+K_{2}}
$$

The conversion itself is simply $m_{01}=A E$ (and $\left.m_{10}=1-A E\right)$. With some algebraic manipulation of Eqs. (7)-(21), we obtain the relations for the effect of dispersed phase mixing:

$$
\begin{aligned}
& \gamma_{20}= \\
& \frac{1-A+2 A(1-E)(1-A E)+(1-A)(1-A E)^{2}(I / 2)}{(1-A E)^{2}(1+A+(1-A)(I / 2))} \\
& \gamma_{02}=\frac{2+(1-A)(I / 2)}{2 A+(1-A)[1+(I / 2)]} \\
& \gamma_{11}=\frac{A(1-E)+(1-A E)[1+(1-A)(I / 2)]}{(1-A E)[1+A+(1-A)(I / 2)]}
\end{aligned}
$$

Interestingly enough the mixing parameter for these associated second order reactions appears as I/2, a definition preferred by SPIELMAN and LEVENSPIEL [2], as in the Monte Carlo procedure it represents the number of coalescences occurring in the reactor during the mean time between drop entries. However, $I / 2$ would not be the form if we wanted higher moment relations. The parameter $I$ is preferred here as it represents the volume fraction of the dispersed phase entering into coalescences per dispersed phase residence time.

Equations (22)-(24) are shown in Figs. 2-4. Equation (7) for $\gamma_{02}$ does not depend on $E$. We see

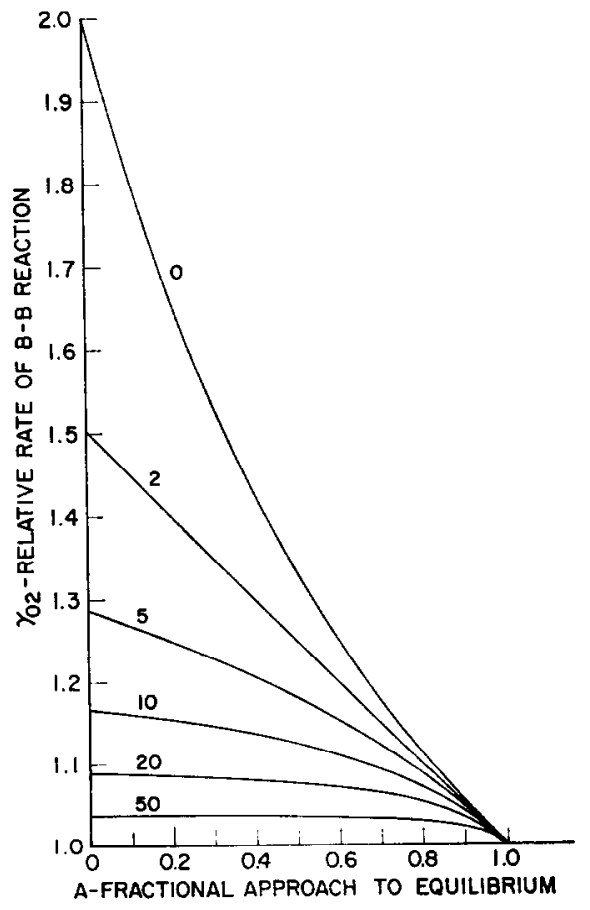

Fig. 2. Rate of reaction $\mathbf{B}+\mathbf{B}$ relative to rate at $I=\infty$ vs. fractional approach to equilibrium. 
R. L. CURL

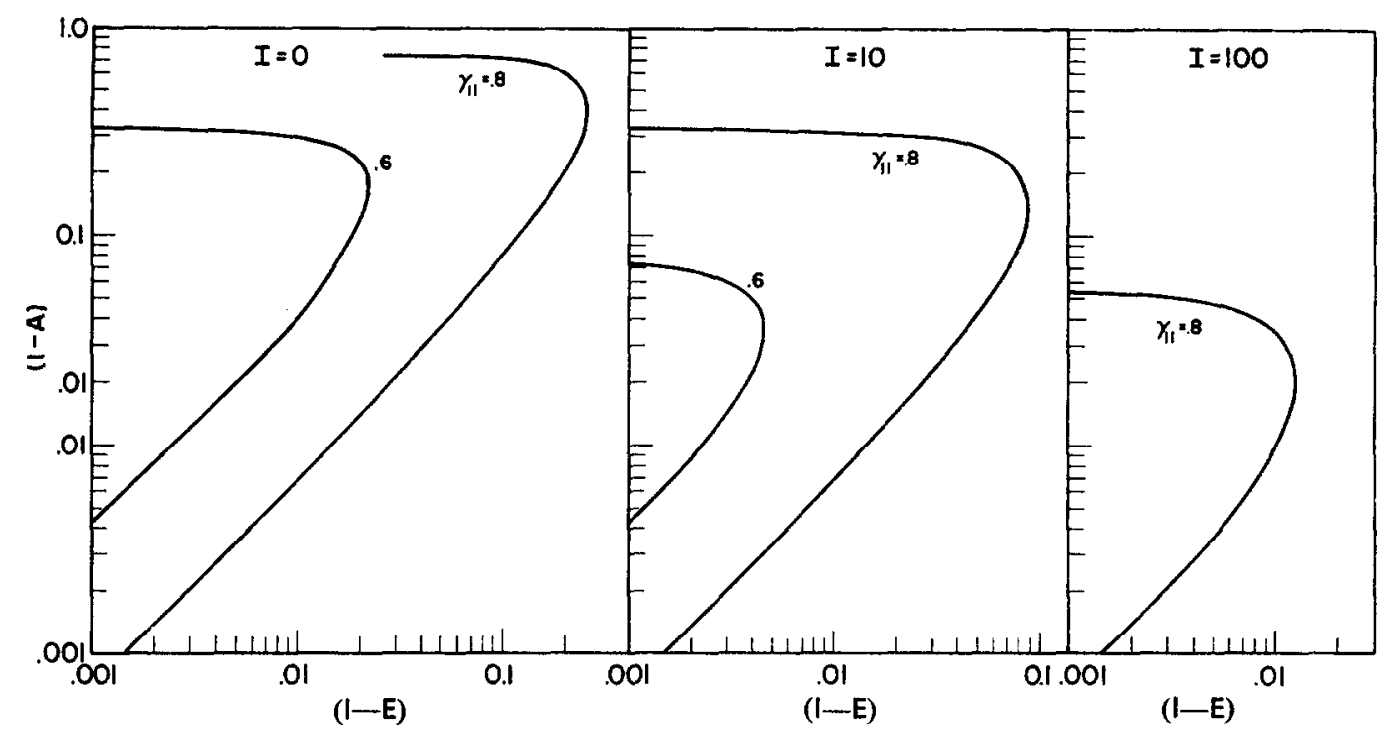

Fig. 3. Rate of reaction $\mathbf{A}+\mathbf{B}$ relative to rate at $I=\infty$. Contours are for constant $\gamma_{11}$. Values at high conver sion are included by plotting parametrically vs. $1-A$ and $1-E$.

that dispersed phase mixing can produce a twofold difference in the relative rate of the second order reaction $\mathbf{B}+\mathbf{B}$, increasing $I$ decreasing the importance of this reaction (the "usual" direction for the effect of mixing in a second order reaction). This dependence is also found for the reaction $\mathbf{A}+\mathbf{A}$, although here $\gamma_{20}$ may become very large at high conversions (similar to the irreversible second order reaction results of SPIELMAN and LEVENSPIEL [2]). However the relative rate of reaction $\mathbf{A}+\mathbf{B}$ is increased by dispersed phase mixing, as shown by $\gamma_{11}$ increasing, within the limits of 0.5 to 1.0 , with increasing $I$. This is qualitatively similar to an $\mathbf{A}+$ $\mathbf{B}$ reaction ( $\mathbf{A}+\mathbf{B}$ separated in the feed) analyzed by SPIELMAN and LEVENSPIEL [7].

As might be expected $\gamma_{20}{ }^{-1}$, with $E=1.0$, becomes asymptotic to the "performance ratio" of Spielman and Levenspiel for a second order reaction, at low conversions. Incidentally, with $I=0$, the present $\gamma_{20}$ is nearly identical to the "performance ratio" for a reactor with an irreversible zeroorder reaction. There may be an intuitive argument for this, but it is not known to the author.

We see that it would be advisable to increase dispersed phase mixing if the reactions $\mathbf{A}+\mathbf{A}$ or $\mathbf{B}+\mathbf{B}$ are the undesired side reactions, although the effect on the latter is small; while we would wish to maintain a segregated dispersed phase if $\mathbf{A}+\mathbf{B}$ were the undesired reaction. We also see that in all cases extremely large mixing rates are necessary to approach the "completely mixed" situation, especially at high conversions.

To what extent a particular rate can be obtained depends on the fluid phases, reactor geometry and mixing rate, as shown by MILLER et al. [5], MADDEN and DAMERELL [6], and GROOTHUIS and ZUIDERWEG [7].

\section{NotATION}

$A$ fractional approach to equilibrium, $C_{B} / C_{B e}$

$C$ concentration of reactant in drop

$\bar{C}$ mean concentration of reactant over all drops

$E$ equilibrium conversion for first order reaction

$I$ dispersed phase mixing modulus, $\omega_{i} / \omega_{r}$

$k$ reaction rate constant, time ${ }^{-1}$

$K$ reaction modulus, $k / \omega_{r}$

$m_{r} \quad r$ th movement of $p(y), \overline{y^{r}}$

$m_{r r^{\prime}} \quad r, r^{\prime}$ joint moment, $\frac{y^{r}(1-y)^{r^{\prime}}}{m^{\prime}}$

$n$ order of reaction

$p$ probability density distribution of $c, y$ or other variates

$r$ order of moment

$t$ time

$y$ dimensionless concentration, $C_{A} / C_{A O}$

$\alpha$ dummy variable 
Dispersed phase mixing effects on second moments in domınantly first-order, backmix reactors
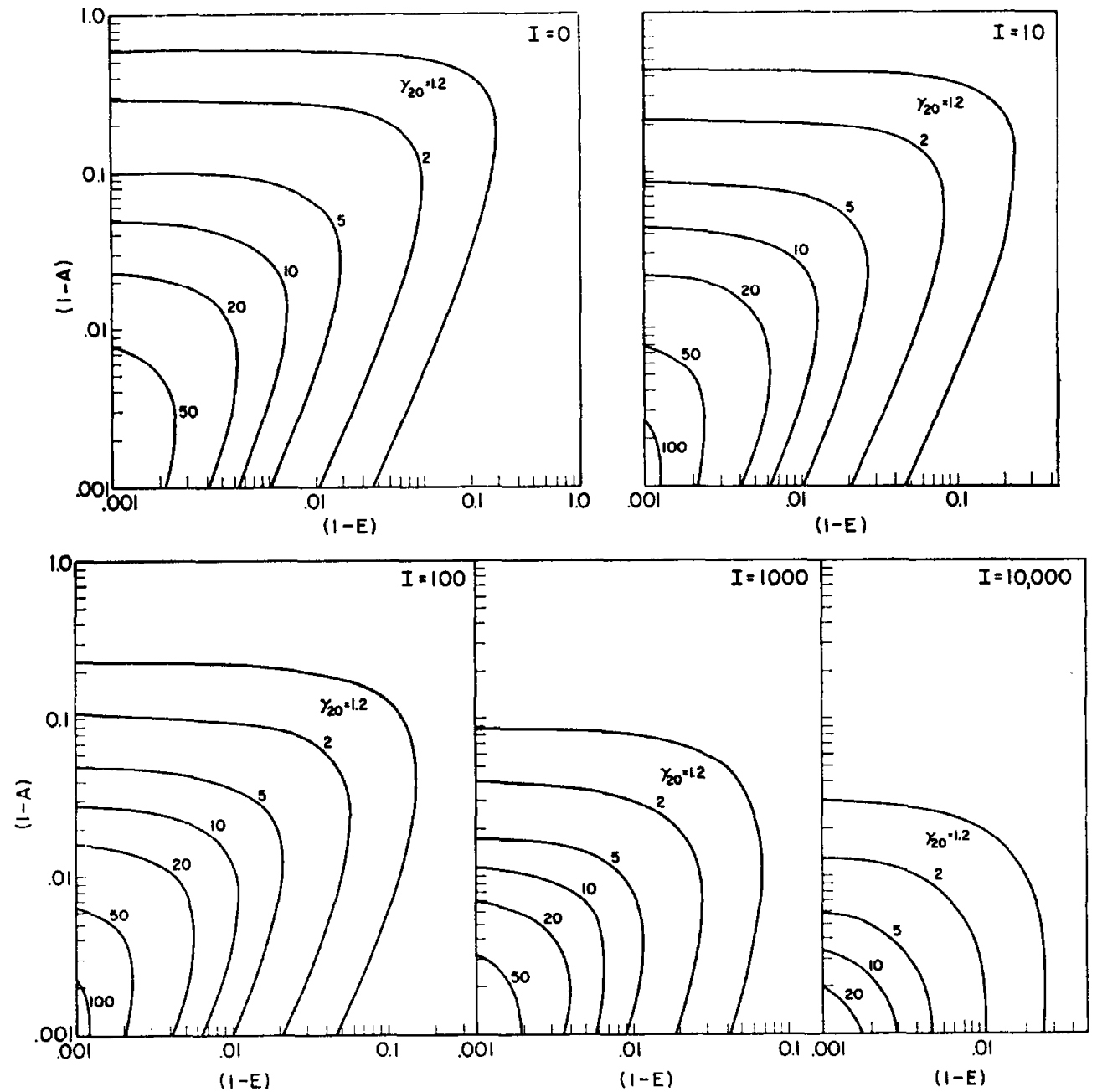

Fig. 4. Rate of reaction $A+B$ rrelative to rate at $I=\infty$. Contours are for constant $\gamma_{20}$.

$\gamma_{r r^{\prime}}$ higher order reaction rates relative to com- Subscripts

plete mixing,

$$
\frac{m_{r r^{\prime}}}{\overline{y^{r}}}
$$

$\omega_{r}$ residence frequency for dispersed phase, time $^{-1}$

$\omega_{i}$ dispersed phase mixing rate, time $e^{-1}$
1,2 forward and reverse reaction, respectively $A, B$ refers to reactants $A$ and $B$

$e$ equilibrium condition

(O) feed condition

\section{Operation}

() mean value.

\section{REFERENCES}

[1] CuRL R. L., A. I. Ch. E. Jl 19639175.

[2] Spielman L. A. and Levenspiel O., Chem. Engng Sci. 196520247.

[3] RIETEMA K., Chem. Engng Sci. 19588 103; Adv. Chem. Engng 19645237.

[4] Hulburt H. M. and Katz S., Chem. Engng Sci. 196419555.

[5] Miller R. S., Ralph J. L., Curl R. L. and Towell G. D., A. I. Ch. E. Jl 19639196.

[6] Madden A. J. and Damerell G. L., A. I. Ch. E. Jl 19628233.

[7] Groothuts H and Zumerweg F. J., Chem. Engng Sci. 1964191963. 


\section{R. L. CURL}

Résumé-L'auteur a employé un modèle idéalisé pour étudier les éffects du dégré de mélange sur la vitesse rélative des réactions simultanées du type $A+A, B+B$, et $A+B$, dans le cas où la réaction $A \rightleftharpoons B$, du supposée du prémier ordre et réversible, est la plus rapide. L'on démontre ainsi la possibilité d'un éffet de mélange prononcé sur la vitesse de la Reaction $A+A$, tandis que l'éffet sur les réactions $B+B$ et $A+B$ peut s'exprimer par un facteur qui m'est jamais plus grand que deux.

Les résultats de cet étude pourraient s'employer pour estimer l'éffet du dégré de mélange sur les réactions du deuxiéme ordre des sous-produits, dont des traces ont un éffet important. En outre, ils représents la première solution analytique des équations de mélange-reaction.

Zusammenfassung-Ein idealisiertes Modell für das Mischen disperser Phasen dient zur Auffindung der Relativgeschwindigkeiten $\operatorname{der}$ Reaktionen $A+A, B+B$ und $A+B$, wenn die Reaktion $A \rightleftharpoons B$ vorherrschend, erster Ordnung und umkehrbar ist. Ein möglichst grosser Mischeffekt auf die Reaktion $A+A$ wird dargestellt, während der Effekt auf $B+B$ oder $A+B$ nie grösser als ein Faktor zwei ist.

Die Ergebnisse sind zur Abschätzung des Mischeffekts auf Nebenproduktreaktionen zweiter Ordnung anwendbar, welchewenn zu einem geringen Mass vorhanden-bedeutend sind. Darüberhinaus stellt die erste analytische Lösung der Mischreaktionsgleichung dar. 